\title{
Frequency domain fluorescence microspectrometry: Application to cellular uptake and drug distribution
}

\author{
Petr Praus ${ }^{a, *}$, Eva Kočišováa ${ }^{a}$, Peter Mojzeš ${ }^{a}$, Josef Štěpánek ${ }^{a}$, Franck Sureau ${ }^{b}$ and \\ Pierre-Yves Turpin ${ }^{\mathrm{b}}$ \\ ${ }^{a}$ Charles University in Prague, Faculty of Mathematics and Physics, Prague, Czech Republic \\ ${ }^{\mathrm{b}}$ Acides Nucleiques and Biophotonique, Evry Cedex, France
}

\begin{abstract}
Time-resolved confocal microspectrofluorometry and fluorescence microimaging were used to monitor how the model antisense oligonucleotide is transported into $3 \mathrm{~T} 3$ living cells and distributed inside them. Phosphorothioate analog of 15-mer oligothymidylate labeled by ATTO 425 was complexed with Zn(II) 5,10,15,20-tetrakis(4-N-methylpyridyl) porphyrin as an uptake-mediating agent. Homodyne phase-resolved technique based on a high frequency analog modulation of both exciting diode laser and detector image intensifier was used for time-resolved measurements. Decay-time data obtained within a broad range spectral region have provided unique information about the fate of both fluorophores inside the cell.
\end{abstract}

Keywords: Fluorescence lifetime, oligonucleotide, porphyrin, microspectrofluorometry, cellular uptake

\section{Introduction}

Methods of fluorescence microscopy are widely used in cellular studies employing natural intracellular chromophores $(\mathrm{NAD}(\mathrm{H})$, flavin, porphyrin), synthetic fluorescent dyes (ionic probes, organelle and structure markers), or endogenous fluorescent fusion proteins (GFP, YFP, CFP, etc.) $[2,6,12,13]$. These applications include also monitoring of the cellular uptake and intracellular interaction of exogenous fluorescent or fluorescently labeled compounds such as antitumoral or photosensitizing drugs $[3,4,8,9]$. Standard microscopic imaging based on the fluorescence intensity measurement at certain wavelength may not, however, be suitable to distinguish between different fluorophores whose emission spectra are highly overlapped. Determination of fluorescence lifetimes over the proper spectral region may help not only to distinguish individual components in fluorescence emission of a fluorescent mixture, but also to provide useful information about interactions in intracellular environment.

In order to gain lifetime characteristics from multifluorophore molecular systems in living cells, we have build up a homodyne time-resolved microfluorometer with a fully modulated short wavelength excitation and a broad spectral window. It enables simultaneous spectral and lifetime analysis either from a single subcellular spot in a confocal mode or from a wide field area selected inside the single cell.

\footnotetext{
${ }^{*}$ Corresponding author: Petr Praus, Charles University in Prague, Faculty of Mathematics and Physics, Ke Karlovu 3, Prague 2, CZ-121 16, Czech Republic. Tel.: +420 2219 11474; Fax: +420 2492 2797; E-mail: praus @ karlov.mff.cuni.cz.
} 


\section{Experimental set-up}

Our fluorescence lifetime microspectrofluorometer (see Fig. 1) is based on a phase modulation principle [11]. Two synchronized frequency synthesizers generate the same modulation frequency that is used to modulate the excitation light and the optical detector gain (homodyne method). A variable phase adjustment of the slave synthesizer is used to change the phase shift between the light source modulation and the image detector. Broad spectral detection window ( $375 \mathrm{~nm}$ wide) is chosen to cover both the excitation wavelength, detected via elastic scattering, and the fluorescence emission. From at least three spectral images acquired at different phase shifts, the phase difference of the fluorescence signal from excitation and consequently the fluorescence lifetime can be determined for all emission wavelengths. To resolve multiple components of the fluorescence lifetime, the measurements have to be performed several times using different modulation frequencies. Spectrum acquisition time is within an interval of several minutes at each frequency to get datasets enabling lifetime resolution of $\pm 0.1 \mathrm{~ns}$.

Laser Diode Module (Omicron LDM 442.50.A350) with $50 \mathrm{~mW}$ output is used for excitation at $442 \mathrm{~nm}$ wavelength. The most severe limit for the excitation power is a danger of the sample photobleaching [1,5,7]. Hence, the $50 \mathrm{~mW}$ output power of the laser is attenuated by neutral density filters to the level from 1 to tens $\mu \mathrm{W}$ at the sample. Analog intensity modulation of the laser is obtained by direct coupling of the output sine wave of the Master synthesizer (model 2025 from IFR) to the laser diode excitation module. A laser-beam clean-up filter (LD01-439/8, Semrock, NY, USA) is used to suppress the green fluorescence emission of the laser diode.

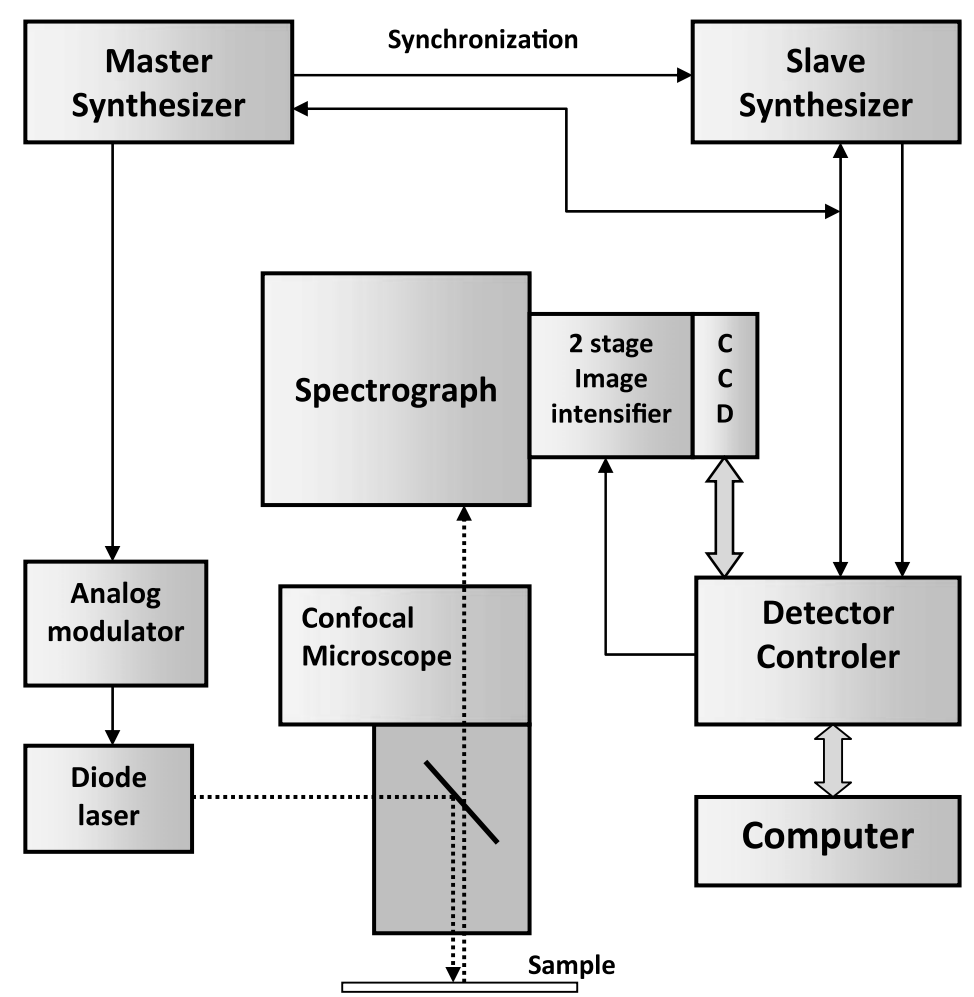

Fig. 1. Block diagram of the experimental set-up of homodyne time-resolved microfluorometer with a broad spectral window. 
Confocal epi-fluorescence upright microscope (Zeiss UMSP-80) is used with a high-numericalaperture water-immersion $63 \times$ objective (Zeiss Neofluar, NA 1.2). Fluorescence signal collected from the pinhole is focused on the entrance slit of the Jobin-Yvon HR640 spectrograph equipped with a 100 line/mm grating, which enables to direct whole $375 \mathrm{~nm}$ spectral region on a $25 \mathrm{~mm}$ diameter photocathode of the image intensifier. This high frequency gain modulated (5-200 MHz) image intensifier (Lambert Instrument, Netherlands) is optically coupled with CCD detector $(1024 \times 1024$ pixels). The CCD controller is connected to the control computer via USB communication interface. The control computer also directly drives both high frequency synthesizers (modulation frequency, the phase and the amplitude of the output signal) via RS232C interface.

The LIFLIM software (Lambert Instruments) is used for the setup control and data acquisition. A special data conversion program (PHR) has been written to calculate the phase shift and the demodulation spectral dependence of the fluorescence signal from the acquired data. Subsequent lifetime determination and spectral decomposition is performed by using a global fitting procedure.

\section{Sample preparation}

The modified oligonucleotide, phosphorothioate oligothymidylate (15-mer), labeled by ATTO 425 at the $3^{\prime}$-end (ATTO-dT 15 ) was synthesized by Biomers, Germany. Chloride salt of $\mathrm{Zn}$ (II) 5,10,15,20tetrakis(4-N-methylpyridyl) porphyrin (ZnTMPyP) was acquired from Frontier Scientific, USA. Living adhesive cell line (3T3 mouse fibroblasts) was cultured as monolayers in $25 \mathrm{~cm}^{2}$ flask in a humidified 5\% $\mathrm{CO}_{2}$ atmosphere at $37^{\circ} \mathrm{C}$, in Dulbecco's Modified Eagle's Medium (DMEM). DMEM was supplemented with $10 \%$ fetal calf serum, $2 \mathrm{mM} \mathrm{L}$-glutamine, streptomycin $(0.1 \mathrm{mg} / \mathrm{ml})$ and penicillin $(100 \mathrm{U} / \mathrm{ml})$, all purchased from Biomedia, France.

Cells were carried to Petri dishes ( $35 \mathrm{~mm}$ in diameter) and subcultured there for $48 \mathrm{~h}$ before microspectrofluorometric experiments. Prior to the experiments, the cells were overnight treated with added solution of oligonucleotide/porphyrin complex. This solution was prepared by gently mixing $60 \mu \mathrm{l}$ of ZnTMPyP stock solution $\left(2.8 \times 10^{-4} \mathrm{M}\right)$ with $30 \mu$ l oligonucleotide stock solution $\left(3.3 \times 10^{-4} \mathrm{M}\right)$ for 15 min.

\section{Experimental results}

As shown in [10], the combination of fluorescence imaging and time-resolved microspectrofluorometry represents promising tool for reliable monitoring of the penetration, distribution and post-delivery fate of the labeled oligonucleotides inside the living cells. Fluorescence imaging can readily confirm successful penetration and shows subsequent distribution of the fluorophore in cellular compartments. On the other hand, the frequency domain lifetime analysis can be used as convenient method to verify integrity of the labeled molecules, stability of their complexes with delivery agents as well as to study their interaction with subcellular structures [11].

In the present study, cellular uptake of ATTO- $\mathrm{dT}_{15}$ oligonucleotides was mediated by fluorescent ZnTMPyP. The fluorescence microimaging proved presence of labeled oligonucleotide and ZnTMPyP in cellular nucleus (figure not shown). Afterwards time-resolved microfluorescence spectra were collected from the cellular nucleus. Fluorescence signal of the two fluorophores was excited by $442 \mathrm{~nm}$ laser line that is close to their excitation maxima (both $436 \mathrm{~nm}$ ). Obtained two-fluorophore experimental 


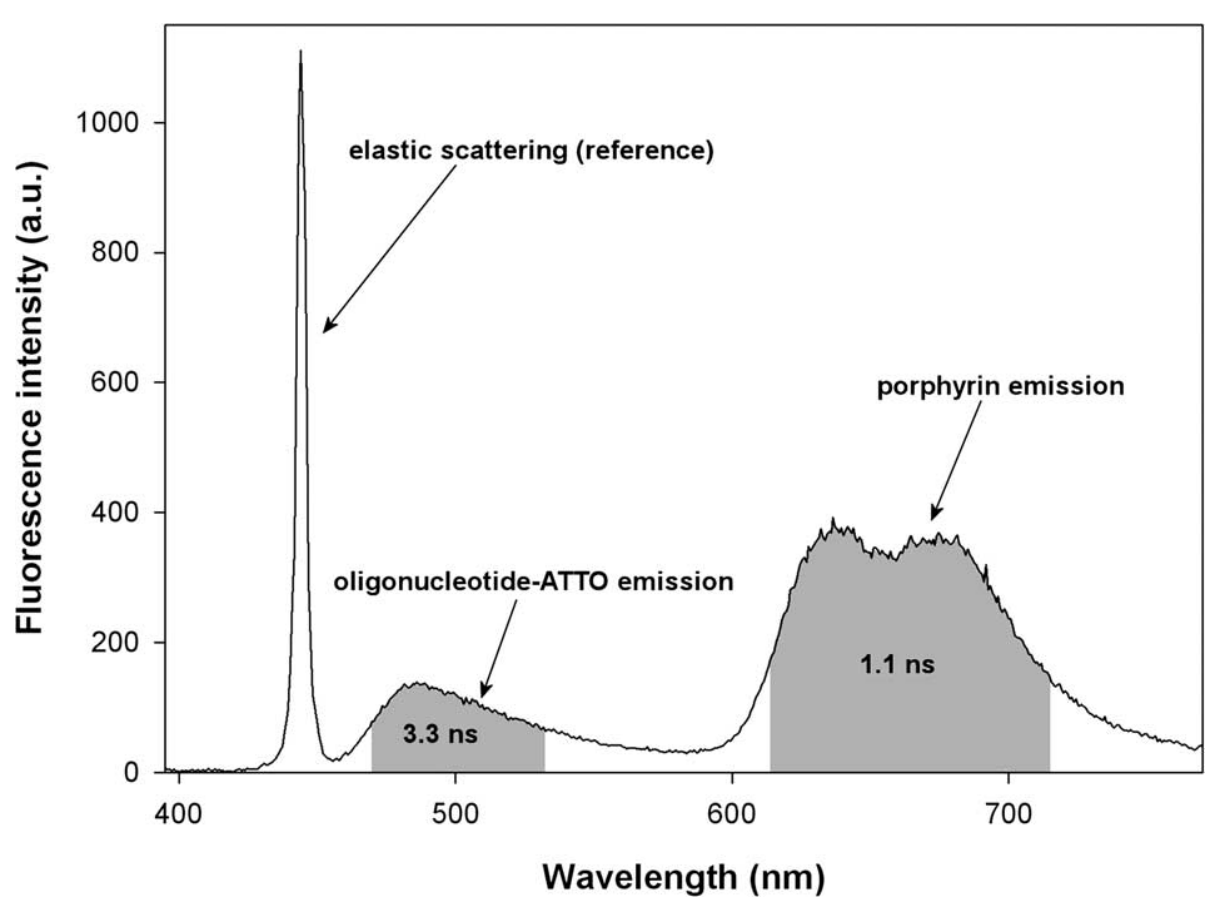

Fig. 2. Original lifetime-resolved spectrum taken from the cellular nucleus of the $3 \mathrm{~T} 3$ mouse fibroblast treated with complex of ATTO labeled oligonucleotide and cationic porphyrin (ATTO-dT $15 / \mathrm{ZnTMPyP}_{\text {). }}$

spectrum is shown in Fig. 2, ATTO-dT 15 and ZnTMPyP fluorescence contributions are clearly separated spectrally ( $\sim 485$ and $\sim 640 / 680 \mathrm{~nm}$, respectively). Elastic scattering peak serves as a zero lifetime reference. As one can see, labeled oligonucleotide and ZnTMPyP contributions are clearly separated. Global analysis has provided two lifetimes: ATTO- $\mathrm{dT}_{15}$ fluorescence can be characterized by a single lifetime of $3.3 \mathrm{~ns}$ and porphyrin fluorescence possesses also a single lifetime but shorter - nearly $1.1 \mathrm{~ns}$. The lifetime of ATTO- $\mathrm{dT}_{15}$ in the cellular nucleus is shortened in comparison with solution (3.6 ns). The porphyrin lifetime also exhibits some shortening after the delivery process (1.7 ns when ATTO-dT 15 /ZnTMPyP complex is in solution). Single lifetime of ZnTMPyP in the nucleus indicates that all porphyrin molecules remain in the complexes with oligonucleotide, in contrast to our previous experiments with complexes of rhodamine-labeled oligonucleotides and free-base $\mathrm{H}_{2}$ TMPyP [10], when two distinct lifetimes of $\mathrm{H}_{2} \mathrm{TMPyP}$ indicated its partial dissociation from the oligonucleotide inside the nucleus [10]. Lifetime shortenings of both fluorophores in ATTO-d $\mathrm{T}_{15} / \mathrm{ZnTMPyP}$ complex probably indicate non-covalent interactions in nuclear environment.

\section{Conclusion}

The build-up homodyne time-resolved microspectrofluorometer with broad spectral window was demonstrated as an efficient tool for monitoring multifluorophore molecular systems inside living cells. Complex of fluorescently labeled phosphorothioate homooligonucleotide with cationic porphyrin ATTO-dT $15 / Z n T M P y P$ was found to penetrate successfully into living cells and to be accumulated in the cellular nucleus without dissociation. 


\section{Acknowledgement}

Financial support from the Ministry of Education of the Czech Republic (MSM0021620835) is gratefully acknowledged.

\section{References}

[1] D.M. Benson, J. Bryan, A.L. Plant, A.M. Gotto Jr. and L.C. Smith, Digital imaging fluorescence microscopy: spatial heterogeneity of photobleaching rate constants in individual cells, J. Cell Biol. 100 (1985), 1309-1323.

[2] D.M. Chudakov, S. Lukyanov and K.A. Lukyanov, Fluorescent proteins as a toolkit for in vivo imaging, Trends Biotechnol. 23 (2005), 605-613.

[3] R.J. Errington, S.M. Ameer-Beg, B. Vojnovic, L.H. Patterson, M. Zloh and P.J. Smith, Advanced microscopy solutions for monitoring the kinetics and dynamics of drug-DNA targeting in living cells, Adv. Drug Deliv. Rev. 57 (2005), $153-167$.

[4] E.A. Forssen, R. Malé-Brune, J.P. Adler-Moore, M.J. Lee, P.G. Schmidt, T.B. Krasieva, S. Shimizu and B.J. Tromberg, Fluorescence imaging studies for the disposition of daunorubicin liposomes (DaunoXome) within tumor tissue, Cancer Res. 56 (1996), 2066-2075.

[5] R.A. Hoebe, C.H. van Oven, T.W. Gadella Jr., P.B. Dhonukshe, C.J. van Noorden and E.M. Manders, Controlled lightexposure microscopy reduces photobleaching and phototoxicity in fluorescence live-cell imaging, Nat. Biotechnol. 25 (2007), 249-253.

[6] A. Müller-Taubenberger, Application of fluorescent protein tags as reporters in live-cell imaging studies, Methods Mol. Biol. 346 (2006), 229-246.

[7] T. Nishigaki, C.D. Wood, K. Shiba, S.A. Baba and A. Darszon, Stroboscopic illumination using light-emitting diodes reduces phototoxicity in fluorescence cell imaging, Biotechniques 41 (2006), 191-197.

[8] M. Olivo and B.C. Wilson, Mapping ALA-induced PPIX fluorescence in normal brain and brain tumor using confocal fluorescence microscopy, Int. J. Oncol. 25 (2004), 37-45.

[9] I.A. Patito, C. Rothmann and Z. Malik, Nuclear transport of photosensitizers during photosensitization and oxidative stress, Biol. Cell 93 (2001), 285-291.

[10] P. Praus, E. Kočišová, P. Mojzeš, J. Štěpánek, F. Sureau and P.-Y. Turpin, Time-resolved microspectrofluorimetry and fluorescence imaging techniques: study of porphyrin-mediated cellular uptake of oligonucleotides, Ann. N. Y. Acad. Sci. 1130 (2008), 117-121.

[11] P. Praus, E. Kočišová, O. Seksek, F. Sureau, J. Štěpánek and P.-Y. Turpin, Advanced microfluorescence methods in monitoring intracellular uptake of antisense oligonucleotides, Curr. Org. Chem. 11 (2007), 515-527.

[12] J. Zhang, R.E. Campbell, A.Y. Ting and R.Y. Tsien, Creating new fluorescent probes for cell biology, Nat. Mol. Cell Biol. 3 (2002), 906-918.

[13] W.R. Zipfel, R.M. Williams, R. Christie, A.Y. Nikitin, B.T. Hyman and W.W. Webb, Live tissue intrinsic emission microscopy using multiphoton-excited native fluorescence and second harmonic generation, Proc. Natl. Acad. Sci. USA 100 (2003), 7075-7080. 


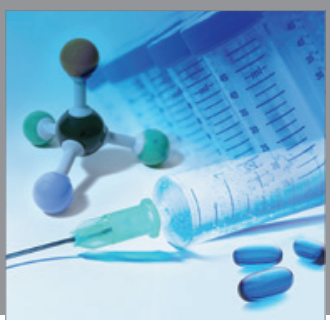

International Journal of

Medicinal Chemistry

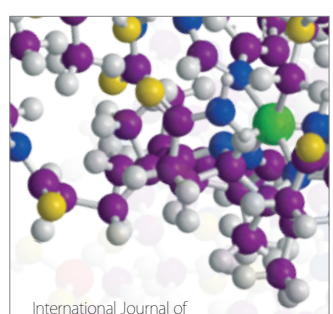

Carbohydrate Chemistry

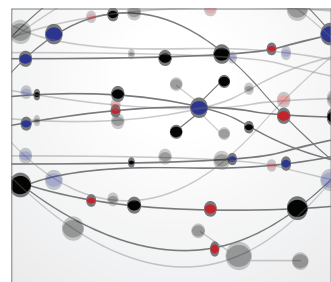

The Scientific World Journal
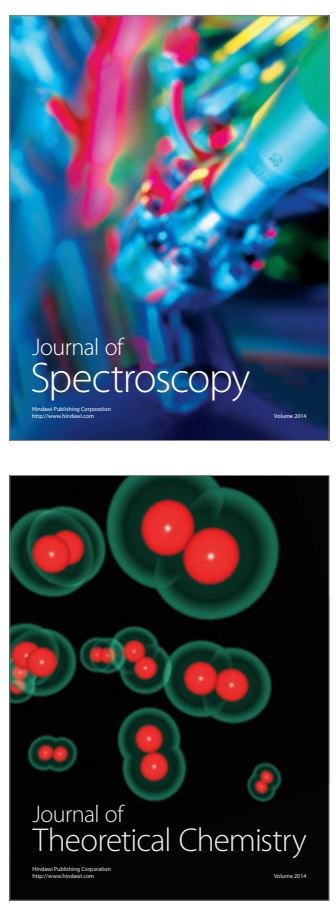
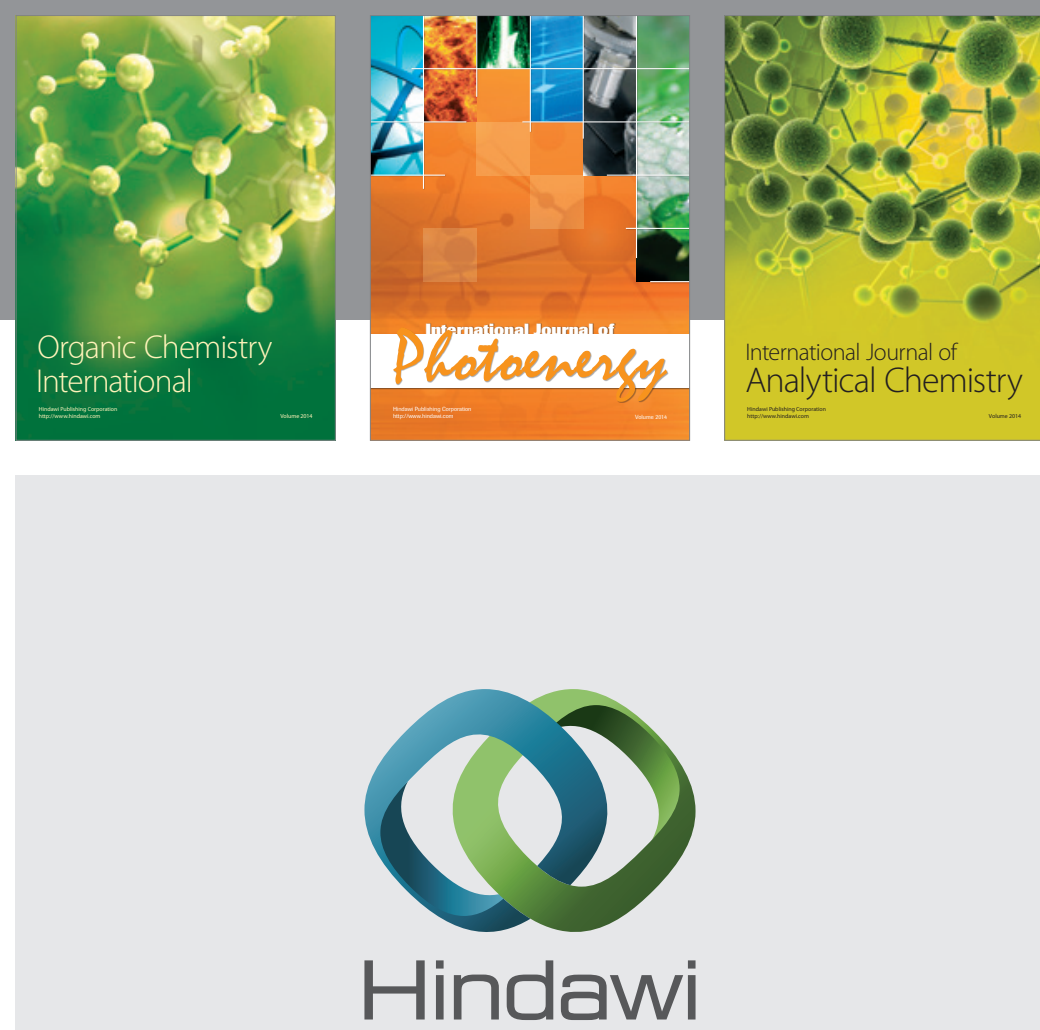

Submit your manuscripts at

http://www.hindawi.com
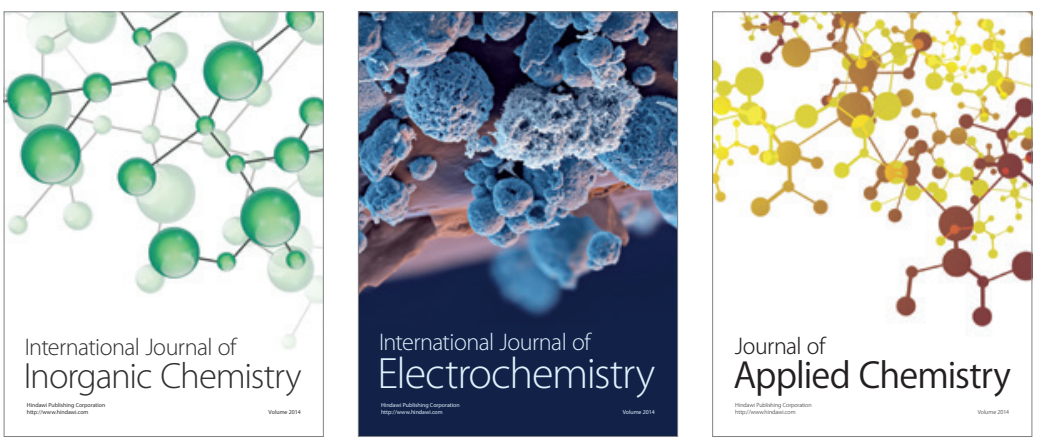

Journal of

Applied Chemistry
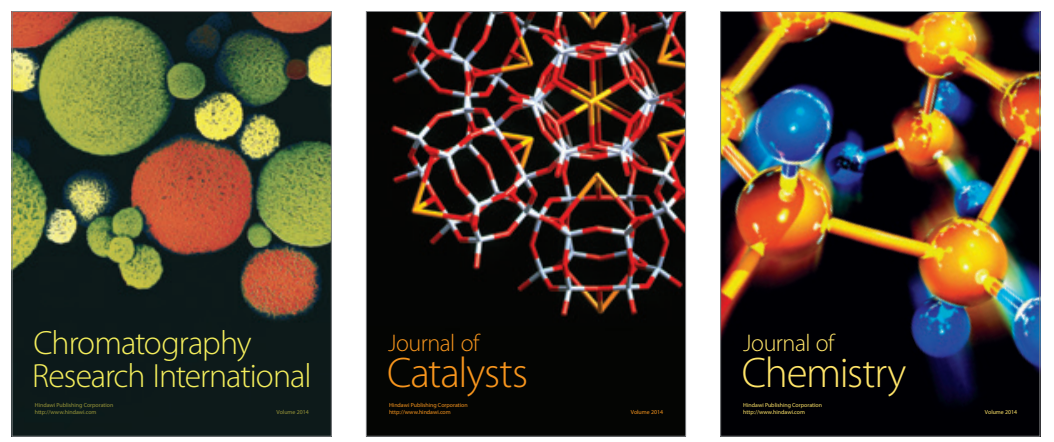
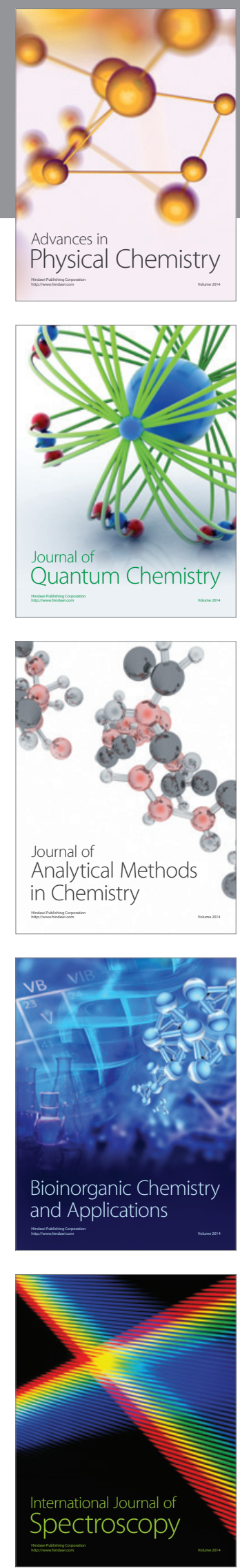